\title{
Tecnologia educacional para mediar práticas educativas sobre alimentação complementar na Amazônia: estudo de validação
}

\author{
Lujhon Rodrigues Viana ${ }^{1}$, Mateus Martins Barreto², Claudia Cristina Pinto Girard³, \\ Elizabeth Teixeira ${ }^{4}$
}

enf.lujhonviana@mail.com, enfmateus2@gmail.com, claudiarupali@gmail.com, etfelipe@ hotmail.com

\begin{abstract}
${ }^{1}$ Universidade do Estado Pará Campus Tucuruí-PA- UEPA, 68440-0oo Abaetuba-PA- Brasil
${ }^{2}$ Universidade do Estado do Pará Campus Tucuruí-PA-UEPA, 68440-0oo Abaetetuba -PA- Brasil

${ }^{3}$ Universidade do Estado do Pará Campus Tucuruí-PA-UEPA, 68464655 Tucuruí -PA-Brasil

${ }^{4}$ CEULM-ULBRA e Universidade do Estado do Amazônas.
\end{abstract}

DOI: 10.17013/risti.28.29-40

\begin{abstract}
Resumo: O estudo teve como objetivo validar uma cartilha para mediar o ensinoaprendizagem de mães de crianças de seis a doze meses sobre a alimentação complementar. Pesquisa metodológica com abordagem mista, realizada na Universidade do Estado do Pará Campus XIII, no município de Tucuruí -PA. Foram participantes 13 juízes da área sócio humanística e da área da saúde. O instrumento de coleta de dados foi um questionário, aplicado no período de dezembro de 2016 a março de 2017. Para o cálculo do índice de validade de conteúdo foi utilizada a estatística descritiva comportamental e para a análise qualitativa a análise temática de convergência. A média dos escores dos itens analisados correspondeu a um IVC de $92 \%$. O resultado apontou a adequação da tecnologia a também a necessidade de aprimoramento. Considerações finais: A tecnologia educacional foi considerada válida estatisticamente para ser usada pelos profissionais da saúde com o público-alvo.
\end{abstract}

Palavras-chaves: Educação em saúde; tecnologia educacional; atenção básica

\section{Educational technology to mediate educational practices on complementary feeding in the Amazon: validation study}

Abstract: The study aimed to validate a primer to mediate the teaching-learning of mothers of children from six to twelve months on complementary feeding. Methodological research with mixed approach, held at the University of Pará Campus XIII, in the municipality of Tucuruí -PA. Thirteen judges from the sociohumanistic and health area were participants. The data collection instrument was a questionnaire, applied in the period from December 2016 to March 2017. For the calculation of the index of content validity was used descriptive behavioral statistics and for the qualitative analysis the thematic analysis of convergence. 
The mean of the items analyzed corresponded to a CVI of $92 \%$. The result pointed to the suitability of the technology as well as the need for improvement. Final considerations: Educational technology was considered statistically valid for use by health professionals with the target audience.

Keywords: Health education; educational technology; basic care

\section{Introdução}

A alimentação complementar (AC) se caracteriza pelo conjunto de alimentos oferecidos aos lactentes para complementar as diversas qualidades nutricionais do leite materno. A introdução de novos alimentos na alimentação da criança é de extrema importância para o seu crescimento adequado, no entanto, deve-se considerar o período correto para a introdução (Brasil, 2009).

A criança deve ser nutrida somente com o leite materno até o sexto mês, após esse período, outros alimentos devem ser inseridos de forma complementar à amamentação. A inserção gradativa, deve ter preparo especial para a criança, até que a mesma possa receber a alimentação semelhante da família (Brasil, 2009).

Nas últimas décadas, ocorreram mudanças na cultura alimentar dos brasileiros, houve um aumento significativo no consumo de alimentos superprocessados, reduzindo o consumo de alimentos naturais (Helman, 2009).

Na região norte, na abrangência da Amazônia Legal, observa-se ainda a presença de desnutrição crônica, que é um dos agravantes da alimentação insuficiente. Entre os grupos de crianças mais suscetíveis, estão as indígenas e quilombolas (Brasil, 2012).

Nas últimas três décadas observou-se que no Brasil houve o decréscimo da desnutrição infantil, sendo representado por $1,5 \%$ das crianças (Brasil, 2012). Na região norte a desnutrição crônica prossegue em $16 \%$ no programa bolsa família, e em grupos mais suscetíveis como indígenas e quilombolas, o índice de desnutrição é de $26 \%$ e 14,8\% respectivamente (Brasil, 2012).

O sobrepeso e a obesidade infantil são fatores de risco para o desenvolvimento de doenças crônicas não transmissíveis, sendo a principal causa de morbimortalidade no Brasil (Brasil, 2012).

As influências diretas do mercado de trabalho e da globalização fizeram surgir alternativas na indústria alimentícia, favorecendo o acesso a alimentos industrializados e a difusão de gêneros alimentícios geneticamente modificados, instaurando um novo estilo alimentar não saudável e que favorecem o desaparecimento gradativo da cultura alimentar regionalizado (Helman, 2009).

O novo padrão de consumo alimentar influencia na escolha os alimentos que serão ofertados ao lactente a partir de sexto mês de vida, quando a alimentação complementar deve ser iniciada. Até o sexto mês é recomendado o aleitamento materno exclusivo sem incluir qualquer alimento sólidos, líquidos ou pastosos. A alimentação complementar é indicada pois a criança já possui os reflexos de deglutição e lingual, começam a maturação muscular-esquelético para a sustentação da cabeça e o surgimento dos primeiros dentes (Brasil, 2013). 
A Política Nacional de Atenção Integral à Saúde da Criança (PNAISC), tem a finalidade de proteger, promover a saúde da criança, com uma atenção maior na primeira estimulando o aleitamento materno e a $\mathrm{AC}$, com o objetivo de diminuir a morbimortalidade e criando condições propícias para uma boa existência e desenvolvimento, através de uma assistência integral (Brasil, 2015).

A criança tem direito à alimentação, segundo a declaração dos direitos universais e a PNAISC estabelece como ações estratégicas o aleitamento materno e a alimentação complementar saudável.

A educação em saúde é um campo de práticas e de conhecimentos do âmbito da saúde que tem se ocupado mais diretamente com a criação de vínculos entre a ação assistencial e o pensar e fazer cotidiano da população (Brasil, 2007).

No período militar, na década de 60, o sistema de saúde imposto fortalecia o assistencialismo médico e não permitia a prática da educação em saúde com a participação popular (Brasil, 2007), porém a partir da década de 70, novos modelos de educação, mais participativos vem sendo discutidos no contexto social (Freire, 2011).

O termo conscientização é referido como uma necessidade do desvelamento da realidade de forma objetiva crítica e desafiadora pelas massas populares para obter a transformação (Freire, 2011).

Em sua vasta obra este autor defende uma educação problematizadora e libertadora que enfatiza o aprendizado que implica a ação e a reflexão dos homens sobre o mundo, em um processo interativo entre educador-educando e educando-educador, pois ambos aprendem em uma relação dialógica e humanista e que produz o empoderamento (Freire, 2011).

O empoderamento implica na conquista da liberdade, autonomia e superação do estado de subordinação, sendo um processo ativo que é realizado pela própria pessoa, que evolui os indivíduos através de mudanças e ações que a levam a evoluir e se fortalecer. A pessoa empoderada realiza as mudanças necessárias para seu fortalecimento (Valoura, 2005/2006).

É importante que o enfermeiro realize ações de educação em saúde e para tal, necessita compreender o significado do alimento, que é mais que uma fonte de nutrição, mas uma forma de expressar aspectos sociais, religiosos e econômicos (Helman, 2009).

O uso de tecnologias como mediadoras do processo ensino-aprendizagem em ações de educação em saúde, favorece o processo participativo com a comunidade, desde que as necessidades dos atores sociais seja o ponto de partida para a produção. $\mathrm{O}$ uso de tecnologia educacional numa perspectiva emancipatória é não se limitar ao mero uso como um equipamento, mas como o conjunto sistemático de procedimentos que torna possível o planejamento, a execução e a avaliação da educação em saúde e que permite o indivíduo a pensar, refletir, agir, tornando-o sujeito de seu próprio processo existencial (Niestche et al, 2012).

Tais reflexões levaram uma das autoras, entre 2013 a 2015, a produzir e posteriormente validar uma tecnologia do tipo cartilha. A produção foi com base em evidências da literatura e um estudo exploratório descritivo com abordagem qualitativa que teve como objetivo compreender as experiências, perspectivas e expectativas de mães e enfermeiras 
sobre alimentação complementar da criança de seis a doze meses (Girard, 2015; Viana, Barreto, Girard, \& Teixeira, 2018).

A cartilha, denominada "Alimentação Complementar da criança de 06 a 12 meses: O que é importante saber” foi composta por uma capa e 27 páginas, com margens superior e inferior $2,5 \mathrm{~cm}$, esquerda e direita $3 \mathrm{~cm}$. Cada página continha textos e até 06 imagens, totalizando 43 ilustrações ao todo. Quanto ao conteúdo, a cartilha foi organizada em o4 partes assim denominadas: O que é alimentação complementar e higiene alimentar; composição dos grupos dos alimentos; seleção e quantidade de alimentos e receitas para o preparo dos alimentos.

A referida construção emergiu de uma dissertação de mestrado realizada no Curso de Mestrado Associado em Enfermagem da Universidade do Estado do Pará e Universidade Federal do Amazonas (Girard, 2015).

Diante do exposto, tendo como referência a cartilha produzida, buscou-se resposta à seguinte questão de pesquisa: a cartilha sobre alimentação complementar para mães de crianças de seis a doze meses é adequada e válida segundo juízes- especialistas?

Este estudo teve como objetivos: Validar uma cartilha para mediar o ensino-aprendizagem de mães de crianças de seis a doze meses sobre alimentação complementar e verificar se a cartilha é um instrumento estatisticamente válido para ser usado com o público-alvo segundo os juízes- especialistas.

\section{Metodologia}

Trata-se de um estudo de validação de conteúdo, do tipo pesquisa metodológica com abordagem mista. Os estudos metodológicos tratam do desenvolvimento, da validação e da avaliação de ferramentas e métodos de pesquisa (Polit \& Beck, 2011).

O processo de validação ocorreu na Universidade do Estado do Pará Campus XIII, no município de Tucuruí -PA. Tucuruí é um município situado à margem do rio Tocantins, no sudeste do Pará a $420 \mathrm{~km}$ da capital, na Amazônia Legal, com população de área urbana e rural em torno de $\mathbf{1 0 0 . 0 0 0}$ habitantes (Instituto Brasileiro de Geografia e Estatística [IBGE], 2012).

Os participantes da pesquisa foram selecionados por atuarem na atenção básica e também na docência. A opção por esses campos de atuação se deu pelo fato desses locais desenvolverem tanto o ensino-aprendizagem como a assistência à criança.

Os participantes deste estudo foram 13 profissionais, denominados de juízes da área sócio humanística (dois pedagogos, um licenciado em língua portuguesa, um publicitário e um tecnólogo da informação) e juízes da área da saúde (quatro enfermeiros, dois nutricionistas, dois médicos).

Os juízes da área da saúde incluídos atuavam no mínimo cinco anos com o programa saúde da criança e tinham pós-graduação em saúde pública, pediatria ou nutrição; os juízes de outras áreas tinham experiencia na área no mínimo há três anos. Os juízes foram selecionados previamente por análise dos currículos lattes, feita no website Plataforma Lattes. De acordo com os critérios de seleção, frente ao perfil da pesquisa, foram convidados pessoalmente, por e-mail ou contatados por telefone. 
Identificou-se os juízes de outras áreas, utilizando códigos com as 2 letras JO, seguidas de numeração crescente da seguinte forma: $\mathrm{JO}_{1}, \mathrm{JO} 2, \mathrm{JO}_{3}$, JO4 e JO5. Os Juízes da área da saúde foram identificados com as 2 letras JE para enfermeiros, JM para médicos, JN para nutricionistas, seguida de numeração crescente: JE1, JE2, JE3, JE4, JM1, JM2, JN1, JN2. O grupo composto por juízes, ou seja, profissionais, "experts", devem ser efetivamente engajados na área onde está se desenvolvendo o estudo (Scarparo et al, 2012).

O instrumento de coleta de dados foi um questionário, sugerido por Teixeira, (2011) e organizado segundo uma escala de Likert, com itens distribuídos em três blocos, contendo perguntas relacionadas à avaliação do conteúdo da tecnologia educativa.

A escala de Likert é uma técnica que classifica e expressa um ponto de vista sobre um determinado tópico, possuindo várias declarações (itens). Nesta abordagem os participantes podem indicar até que ponto concordavam ou discordavam da declaração (Polit \& Beck, 2011).

O questionário, dividido em 3 blocos, continha os seguintes itens: a) objetivos: correspondia aos propósitos, metas ou fins que se desejava atingir com a utilização da tecnologia educativa; b) estrutura e apresentação: referia-se à forma de apresentar as orientações. Isto incluía sua organização geral, estrutura, estratégia de apresentação, coerência e formatação; c) relevância: referia-se às características que avaliavam o grau de significação do material educativo apresentado.

Os juízes levaram em consideração as pontuações adotadas para o julgamento da TE $(1,2$, 3 ou 4) e seguiram a escala contendo 4 graus de valoração, onde 1 representava totalmente adequada (TA), 2 adequada (A), 3 parcialmente adequada (PA) e 4 inadequada (I). O questionário também oferecia espaços para anotações ou considerações e/ou sugestões de cada participante do estudo. Este estudo foi submetido e aprovado pelo Comitê de ética sob o Parecer CAAE 598707160000051170. A pesquisa foi realizada em etapas, a seguir descritas.

\subsection{Primeira Etapa}

Identificação e convite aos juízes: os especialistas que aceitaram participar da pesquisa assinaram receberam um KIT validação (em um envelope) contendo: carta convite (Apêndice B), exemplar da Tecnologia Educativa, TCLE e Questionário de avaliação da TE. Foi estabelecido um prazo com os juízes para o retorno do instrumento de coleta de dados (KIT validação) de 15 a 20 dias.

\subsection{Segunda Etapa}

Validação de conteúdo: obtenção das respostas que indicavam o nível específico de concordância ou discordância com cada declaração. Obtenção das sugestões e opiniões (Polit \& Beck, 2011).

O primeiro grupo de juízes nomeados "juízes de outras áreas" foram importantes no julgamento referente à organização, processo de formação cultural e transmissão dos saberes; linguagem, grafismo, diagramação, comunicação visual; avaliação da arte, linguagem expressiva e forma de conhecimento; repasse de informações; layouts das apresentações.

O segundo grupo de juízes nomeados "juízes da área da saúde”, foram importantes para o processo de validação do conteúdo específico abordado na TE. 


\subsection{Terceira Etapa}

Análise quantitativa: foi considerado para o cálculo do Índice de Validade de Conteúdo (IVC), concordância mínima de 80\%, (Pasquali, 2009). O IVC foi obtido com a soma das respostas TA e A. Os cálculos foram realizados manualmente. A estatística descritiva permitiu a adequação da representação comportamental dos itens e as médias da concordância.

Na observação da tendência das respostas, se concordantes ou discordantes, a intenção foi verificar a valoração que obteve a maioria das respostas, para que se tornasse possível inferir se houve ou não indicação significativa de discordância em cada um dos blocos do formulário.

Reagrupou-se as opções de respostas de 1,2, 3 e 4 com 1 e $2(+1), 3$ (o) e $4(-1)$. A resposta de cada juiz poderia variar entre -1 e +1 e quanto mais próxima de +1 , maior a concordância entre eles de que o item era pertinente (índice de concordância).

\subsection{Quarta Etapa}

Análise qualitativa: foi realizada mediante análise temática de convergência, considerando o consenso dos comentários, sugestões, pontos de vista e ideias registradas no questionário.

\subsection{Quinta Etapa}

Elaboração da segunda versão da cartilha: consistiu na reconstrução da TE a partir das considerações dos juízes especialistas.

\section{Resultado e discussão}

\subsection{Perfil dos Juízes}

\begin{tabular}{l|l|l|l|l|l|l}
\hline Código & Idade & Graduação & $\begin{array}{l}\text { Tempo de } \\
\text { formação }\end{array}$ & $\begin{array}{l}\text { Tempo de } \\
\text { atuação }\end{array}$ & Titulação & $\begin{array}{l}\text { Tempo de } \\
\text { docência }\end{array}$ \\
\hline JO1 & 39 & Pedagogia & 13 anos & 13 anos & Especialista & 20 anos \\
\hline JO2 & - & Pedagogia & 17 anos & 9 anos & Mestre & 9 anos \\
\hline JO3 & 48 & $\begin{array}{l}\text { Licenciatura em letras } \\
\text { língua portuguesa }\end{array}$ & 20 anos & 20 anos & Especialista & 27 anos \\
\hline JO4 & 36 & Tecnologia da informação & 4 anos & 3 anos & - & - \\
\hline JO5 & 29 & Publicidade e propaganda & 10 anos & 9 anos & Especialista & - \\
\hline
\end{tabular}

Quadro 1 - Perfil dos juízes de outras áreas

Percebe-se que os "juízes de outras áreas” possuíam idade entre 29 a 48 anos, com formação no tempo mínimo de 4 anos e no máximo 20 anos. O tempo de atuação na área entre 3 a 20 anos. O juiz com maior titulação era mestre. O número total de juízes desse grupo foram 5 e 60\% atuavam na docência. 


\begin{tabular}{l|c|c|c|c|c}
\hline Código & Idade & Graduação & Tempo de formação & Tempo de atuação & Titulação \\
\hline JE1 & - & Enfermagem & 13 anos & 13 anos & Especialista \\
\hline JE2 & 43 & Enfermagem & 18 anos & 15 anos & Mestre \\
\hline JE3 & 46 & Enfermagem & 21 anos & 21 anos & Especialista \\
\hline JE4 & - & Enfermagem & 21 anos & 17 anos & Especialista \\
\hline JM1 & 41 & Medicina & 18 anos & Enos & Especialista \\
\hline JM2 & - & Medicina & 30 anos & Especialista \\
\hline JN1 & 41 & Nutrição & 19 anos & - & Especialista \\
\hline JN2 & 49 & Nutrição & 26 anos & Especialista \\
\hline
\end{tabular}

Quadro 2 - Perfil dos juízes da área da saúde

Constata-se que os "juízes da área da saúde” têm idade entre 41 a 49 anos. Em relação ao tempo de formação, dos 8 juízes, 4 se se graduaram entre 13- 19 anos (50\%), e 4 entre há 21- 30 anos (50\%). Quanto à titulação e tempo de atuação, 3 profissionais são especialistas em pediatria (37,50\%), sendo 1 enfermeiro e 2 médicos; 2 destes profissionais atuam na área entre 5- 15 anos.

Percebe-se que 4 juízes são especialistas em estratégia de saúde da família e/ou saúde pública (50\%), sendo 1 nutricionista e 3 enfermeiros. Os 2 juízes nutricionistas têm especialização em clínicas (25\%), sendo que um atua na área há 19 anos e um não respondeu quanto ao tempo de atuação.

O perfil dos juízes quando a pós-graduação aponta que 12,50\% têm formação strictusensu e 100\% lato-sensu; o maior grau é de mestrado, o que evidencia a possibilidade de uma avaliação afirmada num olhar teórico-científico-metodológico.

Notou-se predomínio de juízes com experiência e especializados na área da pediatria, saúde da família e nutrição, o que favoreceu o processo de validação no que concerne ao discernimento sobre o conteúdo da tecnologia educativa.

\subsection{Blocos Avaliados}

\subsubsection{Bloco Objetivo}

Do total de juízes, 9 decidiram por assinalar e comentar e 4 apenas assinalar. Assim, verificou-se os seguintes resultados: 46 (70,76\%) assinalaram totalmente adequado, 14 (21,53\%) adequado, 5 (7,69\%) parcialmente adequado, e nenhum assinalou inadequado. Dos 65 itens avaliados, 60 foram considerados TA e A, obtendo-se um índice de validade de conteúdo parcial de 92,29\%.

Do total de juízes, 5 sugeriram ajustes conforme indicam algumas falas: a) "De modo geral a cartilha vem somar conhecimentos para diversas áreas e públicos... a didática é clara (Jo4)”; b) “As orientações são compatíveis com orientações pediátricas em todos os aspectos (JM1)”; c) “Considero de suma importância o conteúdo deste material educativo... ajuda auxiliando aos pais e responsáveis na alimentação dessa faixa etária (Jo3)”; d) "Confere aceitação tornando assim a cartilha apta quantos aos objetivos proposto (JE2)". 
As falas demonstram que a TE poderá contribuir no processo educativo sobre alimentação complementar, auxiliando profissionais, pais e cuidadores no momento da introdução de novos alimentos.

Segundo Cunha et al (2017), a tecnologia é o resultado de processos implementados a partir da experiência e pesquisa cotidiana, para o desenvolvimento de um conjunto de conhecimentos científicos, construindo ou não produtos de materiais, com o objetivo de provocar intervenções para uma determinada situação prática. A aplicação de tais recursos pode potencializar o planejamento da educação em saúde.

A educação em saúde é um campo de ensino com trocas de experiencias, cujo interesse é incorporação das ações que promovam a saúde (Miranda \& Malagutti, 2010). No Brasil a educação em saúde vem sendo utilizada desde a década de 1920. Nesse período, os profissionais utilizavam métodos de ensino verticalizados, para combater e controlar doenças transmissíveis.

Atualmente, o modelo de atenção à saúde no Brasil, o Sistema Único de Saúde (SUS), exige uma postura diferente para a educação em saúde. Ao contrário de métodos verticalizados, as ações educativas devem produzir a crítica capaz de suscitar a autonomia dos indivíduos na promoção da sua saúde e da sua comunidade (Freire, 2011).

Para obter mais êxito na educação e saúde é necessário a associação aos meios de comunicação atuais. O uso de tecnologias validadas pode mediar o processo de ensino/ aprendizagem. Segundo Nietsche (2000), a tecnologia serve para gerar conhecimento a serem socializados entre as pessoas, não podem ser resumidas a meros artefatos ou equipamentos que façam a mediação entre o pensamento e a realização das ações. Percebe-se que é necessário que o educador realize um diagnóstico da situação educativa de seus educandos, ou seja conheça a sua realidade, respeite seus saberes, desta maneira poderá realizar educação em saúde. O uso de tecnologias validadas pode favorecer a troca de saberes e a construção de um ensino compartilhado.

\subsubsection{Bloco Estrutura e Apresentação}

O nível de concordância dos juízes nos itens avaliados foi de $104(68,42 \%)$ para totalmente adequado 35 (23,02\%) para adequado, 12 (7,89\%) para parcialmente adequado e 1 (o,65\%) para inadequado.

Dos 152 itens avaliados 139 (91,44\%) foram assinalados como TA e A, enfatizando-se que a TE é válida em relação à sua estrutura e apresentação, obtendo-se um índice de validade de conteúdo parcial de 91, $44 \%(0,9)$.

Dos juízes que realizaram comentários, 7 sugeriram alterações na TE: a) "Faltou uma formatação adequada (Item 2.9)... algumas imagens estavam com tamanhos diferentes e apagadas (Item 2.10" (JO3); b) “Talvez uma capa dura ajudasse na durabilidade do material" (JO4); c) "Sugiro uma reorganização nas gravuras para que tomem um aspecto mais lúdico" (JO1); d) "uma arte infantil ... podendo ser utilizados vários recursos do design gráfico “( $\left.\mathrm{JO}_{5}\right)$.

Percebe-se segundo as falas, que a estrutura visual da tecnologia foi um aspecto muito evidenciado e discutido pelos profissionais que a avaliaram. Houve sugestões sobre a atualização do conteúdo, solicitações de ajustes de ortografia; alteração no tamanho do 
título; solicitação de alteração de papel; solicitação para cooperar com a coerência das informações; solicitação de ajustes na formatação; solicitação de mudanças nas imagens; alterações da capa, design, layout e arte.

A tecnologia educacional é aquela que agrupa meios de auxiliar a formação de uma consciência para a vida saudável (Nietsche, Teixeira \& Medeiros, 2014). O cotidiano da equipe multidisciplinar na atenção básica carece de inovação, para fugir das atividades monótonas, que não impõem o estimulo para a reflexão. Para que haja promoção da saúde há a necessidade de novas intervenções e estratégias de mudança nos modelos tecnoassistenciais, novos saberes e fazeres que ampliem a qualidade de saúde e vida da população, com vista à intervenções educativas que conduzam o público alvo à compreensão do processo saúde-doença (Silva et al, 2008).

A PNAISC estabelece que a criança tem direito à vida e a saúde. A atenção básica se insere nas diretrizes através da promoção e acompanhamento do crescimento e do desenvolvimento integral e através de medidas que estimulem e promovam o aleitamento materno e a alimentação complementar (Brasil, 2015).

É pertinente as considerações dos juízes especialistas, visto que há necessidade de implementação dos recursos na promoção à saúde. Segundo Nietsche (2011), a tecnologia permite uma reestruturação na vida dos sujeitos, porém não implica em mudanças de pensamento se não houver aspectos estéticos, filosóficos, sociais, éticos, morais e espirituais envolvidos.

Em um estudo (Barbosa et al, 2015), descreve-se que as tecnologias educacionais são incontestáveis nas intervenções educativas. As evidências deste estudo revelam que é necessário contextualizar as necessidades do público alvo, e que cabe ao profissional de saúde determinar qual tipo de tecnologia é o mais pertinente. As tecnologias como folhetos, cartilhas, revistas facilitam o acesso às informações.

Em um estudo realizado por Carlos et al (2016), sobre a concepção e avaliação de tecnologia para a promoção da saúde vocal, a estrutura visual do aplicativo foi muito evidenciada entre os que a avaliaram, pois segundo os autores o layout funciona como porta de entrada para que o leitor se interesse pelo conteúdo. Em um estudo de validação de um álbum seriado, os juízes solicitaram alterações do layout da TE. Observa-se que é cada vez mais frequente a necessidade de adequação dos layouts das tecnologias educacionais (Saraiva, Medeiros \& Araújo, 2018).

A educação em saúde deve favorecer a troca de experiências entre educador e educando, e considerar a sua realidade. Um estudo realizado por Mooz, Getz \& Ladner, (2012), sobre educação em saúde, percebeu-se que as informações repassadas aos usuários não eram compreendidas devido a baixa escolaridade dos mesmos. Percebe-se a importância dos profissionais de saúde considerarem as necessidades do público alvo e adequarem as tecnologias de acordo com as necessidades de tais públicos.

\subsubsection{Bloco Relevância}

Observou-se que 43 (72,88\%) assinalaram totalmente adequado, 13 (22,03\%) adequado, 3 $(5,08 \%)$ parcialmente adequado e nenhum assinalou inadequado. Dos 59 itens avaliados, 56 foram assinalados TA e A, obtendo-se um índice de validade de conteúdo parcial de 95\%. 
Os comentários nesse bloco foram: a) "a cartilha é uma forma de conscientizar tanto as mães como profissionais da saúde para uma alimentação saudável nessa faixa etária dos bebês" (JO4); b) "a cartilha tem relevância significativa, e é uma tecnologia leve para educação em saúde, otimizando conteúdos que ajudam a diminuir dúvidas” (JE2); c) "sugiro o uso deste material em postos de saúde, hospitais, clinicas médicas, etc., beneficiando principalmente as crianças” (JE2).

Tais falas demonstram a importância da cartilha para os juízes e sua contribuição à educação em saúde com os pais no que concerne a AC. Percebe-se pelas falas que a tecnologia tem relevância, por se tratar da promoção da alimentação saudável da criança e por possibilitar a reflexão do uso dos alimentos adequados na faixa etária de seis a doze meses.

A alimentação complementar são alimentos que devem ser introduzidos a partir do sexto mês de vida, e conforme a descrição é a complementação do aleitamento materno (Brasil, 2009).

A “chamada neonatal”, pesquisa realizada pelo Ministério da Saúde (Brasil, 2013), com o objetivo de obter informações sobre a qualidade da atenção ao pré-natal, parto, puerpério, morbidade materna e infantil, a partir de mães e crianças menores de um ano, residentes nos municípios participantes do Pacto pela Redução da Mortalidade Infantil no Nordeste e na Amazônia Legal, apontou que o Pará é o estado de maior prevalência em AME em crianças menores de seis meses alcançando índices de 53,5\%, porém o tempo de permanência em AME ocorre em torno de 28 dias; 30\% das crianças no primeiro mês de vida já não recebem AME; tais resultados demonstram que muito cedo ocorre a introdução de outros alimentos nessa região.

Segundo dados da Pesquisa Nacional de Demografia e Saúde da Criança e da mulher (PNDS), 14\% das crianças já são alimentadas com alimentos semi sólidos aumentando essa prevalência para 30\% após o quarto e quinto mês (Brasil, 2006).

No Brasil, a atenção básica apresenta uma modalidade de atenção oriunda das discussões da $8^{a}$ Conferência Nacional da Saúde, dos princípios da constituição de 1988, e do SUS, sistema, que tem como princípios universalidade, equidade e integralidade da assistência.

Segundo Silva et al (2008), esse modelo prioriza a atenção primária com ênfase na promoção da saúde, portanto é relevante o uso de tecnologias validadas, a implantação de um processo educativo que favoreça o uso da vertente educativa progressista e tecnologias educativas que favoreçam as crianças da comunidade amazônica sobre AC.

\section{Considerações finais}

Foi possível inferir que a TE foi validada, atingindo um índice de validade de conteúdo geral de $92 \%$ entre os juízes especialistas.

A produção de TE validadas, na modalidade impressa, podem contribuir na educação em saúde, uma ação de extrema importância, principalmente quando se relaciona à promoção da saúde alimentar de populações das regiões que necessitam melhorar indicadores de saúde relacionados a saúde da criança. 
Os resultados do estudo apontam para a necessidade do uso de TE validadas para mediar a educação entre profissionais da saúde, pais e cuidadores de crianças no momento da transição de novos alimentos.

Em relação aos comentários e sugestões dos juízes, estes contribuíram para o aprimoramento da TE, e os ajustes resultantes facilitaram a produção da $3^{\mathrm{a}}$ versão da cartilha, agora adequada para mediar o agir dos profissionais com mães de crianças de seis a doze meses.

\section{Referências}

Brasil. (2009). Saúde da criança: nutriçãoinfantil: aleitamento materno e alimentação complementar; 2009. Consultado em: htpp:/www.portal.saude.gov.br.

Brasil. (2009). Centro Brasileiro de Análise e planejamento. Pesquisa Nacional de Demografia e Saúde da Criança e da mulher (PNDS). Consultado em: http://bvsms.saude.gov.br/bvs/publicacoes/pnds_crianca_mulher.pdf.

Brasil. (2012). Política Nacional de Alimentação e Nutrição. Brasília: Ministério da Saúde. Consultado em: htpp:/www.portal.saude.gov.br.

Brasil. (2013). Chamada Neonatal: Avaliação da atenção ao pré-natal, ao parto e aos menores de um ano na Amazônia Legal e no Nordeste. Brasil, 2010. Brasília (DF), 2013. Consultado em: htpp//www.bvsms.saude.gov.br

Brasil. (2015). Portaria $N^{o}$ 1.13o, de 5 de agosto de 2015. Consultado em: http:/www.bvsms.saude.gov.br.

Brasil. (2016). Sistema de vigilância alimentar e nutricional, 2016. Consultado em: http:/www.sisvan@.saude.gov.br.

Carlos, D. A. O., Magalhães, T. O., Filho, J. E. V., Silva, R. M., \& Brasil, C. C. P. (2016). Concepção e Avaliação de Tecnologia mHealth para Promoção da Saúde Vocal. RISTI-Revista Ibérica de Sistemas e Tecnologias de Informação, (19), 46-6o.

Cunha, G. H., Galvão, M. T. G., Pinheiro, P. N. C., \& Vieira, N. F. C. (2017). Health literacy for people living with HIV/Aids: an integrative review. Rev Bras Enferm, 70(1), 180-188. Consultado em: http://www.scielo.br.

Cunha, A de L., et al (2015). O professor de Matemática do ensino médio e as tecnologias de informação e comunicação nas escolas públicas estaduais de Goiás. RISTIRevista Ibérica de Sistemas e Tecnologias de Informação, (SPE4), 1-15.

Freire, P., (2011). Pedagogia da autonomia: saberes necessários à prática educativa. (43. ed). São Paulo, SP: Paz e terra.

Girard, C.C.P. (2015). Saberes e práticas de mães e enfermeiros sobre alimentação complementar. Dissertação de mestrado, Universidade do Estado do Pará, Universidade Federal do Amazonas, Belém. Brasil. Consultado em: https://paginas.uepa.br/ppgenf/files/DISSERTAO_CLAUDIA_GIRARD.pdf

Helman, C. G., (2009). Cultura, saúde e doença. (5 ed). Porto Alegre: Artmed. 
Instituto Brasileiro de Geografia e Estatística (2012). Histórico do município de Tucuruí. Consultado em: hppt:/www.ibge.gov.br/.

Mooss, A., Brock-Getz, P., \& Ladner, R. (2013). A relação entre alfabetização em saúde, conhecimento do estado de saúde e crenças sobre transmissão de HIV/AIDS entre clientes de Ryan White em Miami. Health Education Journal, 72 (3), 292-299. Doi:10.1177/0017896912442952.

Miranda, S. M. R., \& Malagutti, W. (2010). Educação em saúde, (p. 312). São Paulo: Phorte.

Nietsche, E. A., Lima, M. G. R., Rodrigues, M. G. S., Teixeira, J. A., Oliveira, B. N. B., Motta, C. A., Gribler, C. S., Gribler, V. M., Lucas, D. D. I., \& Farias, M. K. F. (2012). Tecnologias inovadoras do cuidado em enfermagem. Rev. enferm. UFSM. 2(1), 182-189. Doi: 10.5902/217976923591.

Pasquali, L. (2009). Psicometria. Rev Esc Enferm USP. 43(spe), 992-999. Doi: 0.1590/ Soo80-62342009000500002.

Polit, D. F., \& Beck, C. T. (2011). Fundamentos de Pesquisa em enfermagem: Avaliação de evidências para as práticas da enfermagem. (7 ed). Porto Alegre, RS: Artmed.

Saraiva, N. C. G., Medeiros, C. C. M., \& Araújo, T. L. (2018). Validação em série de álbuns para promoção do controle de peso corporal infantil. Revista Latino-Americana de Enfermagem, 26, (e2998). Sítio web: https://dx.doi.org/10.1590/15188345.2194.2998.

Silva, K. L., Sena, R. R., Gillo, M. J. C., Horta, N. C., \& Prado, P. M. C. (2009). Educação em enfermagem e os desafios para a promoção de saúde. Rev. bras. enferm, 62(1), 86-91. Doi:10.1590/So034-71672009000100013.

Scarparo, A. F., et al. (2012). Reflexões sobre o uso da Técnica Delphi em pesquisas de enfermagem. Sítio web: http://www.periodicos.ufc.br/rene/article/ download/3803/3000. Acesso em o8 abril 2017.

Teixeira, E., \& Mota, V. M. S. S., (2011). Tecnologias educacionais em foco. (1 ${ }^{\text {a }}$ Ed). São Caetano do Sul, SP: Difusão.

United Nations Children's Fund (2016). Nutritional companion to breastfeeding after 6 months. Sítio web: http:/www.unicef.org/programme/breastfeeding/food.htm. Acesso em: 29 fev. 2016.

Valoura, L. C. (2005/2006). Paulo Freire, o educador brasileiro autor do termo Empoderamento, em seu sentido transformador. Sítio web: http:/ www.otics.org/. Acesso em: 12 ago. 2018.

Viana, L. R., Barreto, M. M., Girard, C. C. P., \& Teixeira, E. (2018). Tecnologia Educacional para Mediar a Alimentação Complementar na Amazônia: Estudo de Validação. Em: Anais do $7^{O}$ Congresso Ibero-Americano em Investigação Qualitativa - CIAIQ. Fortaleza, CE, Brasil. 2, 1312-1317. ISBN: 978-972-8914-83-7.

World Health Organization(2000). Collaborative Study Team on the Role of Breastfeeding on the Prevention of Infant Mortality. Lancet, 355 (9202), 451-5. Sítio web: https://www.ncbi.nlm.nih.gov/pubmed/10841125. Acesso em: 05 mai. 2016. 